\title{
Integration of new surgical approaches in Urinary Stress Incontinence after failure of traditional techniques.
}

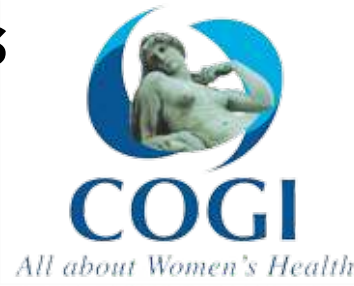

Gabasa-Gorgas L1, Ruiz-Martínez S1 , Procas-Ramón B ${ }^{1}$, Pellicer-Soria AM1, Cabistany AC², Sanz-López A , Bescos-Santana E1, Martínez-Medel J1.

${ }^{1}$ Gynecology, Hospital Clinico Universitario Lozano Blesa. Zaragoza. Spain. Emergency, Hospital Universitario Miguel Servet. Zaragoza. Spain.

\section{Objectives:}

To describe the results in Pelvic floor Unit of a terciary Hospital during 2016, highlighting those that recivided after TOT.

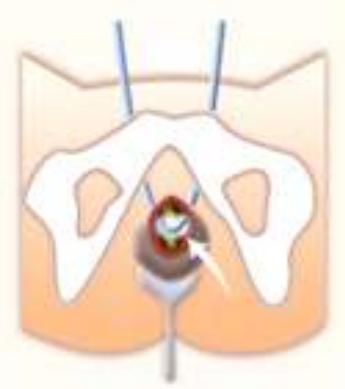

TVT

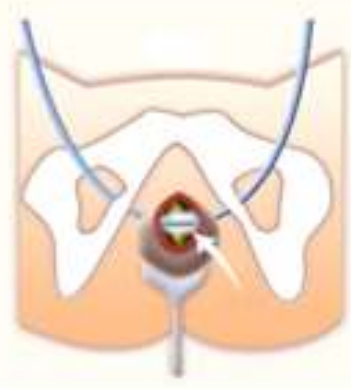

TOT

\section{Methods:}

We did a retrospective study of the patients refered to Pelvic Floor Unit during 2016. We collected the reason for consultation, personal and gineco-obstetrics backgrounds. All of them, did the EPIQ-questionare and a physical exam. After that, a diagnosis was stablished and a treatment was proposed for each patient.

In this study we describe those that present Urinary Stress Incontinence after TOT procedures to perform a personalised surgical treatment.

\section{Results:}

In total, we did 52 TOT procedures and 3 complications.

The first patient, showed stress incontinence one year after the TOT procedure. During the phisical examination, urethra hipermobility was found. We first proposed a Pelvic Floor rehabilitation, but, without improvement, TVT procedure was done. The surgery was succesful. 8 months later the patient is asymptomatic.

The second patient, had 2 surgical operations with TOT procedures. She continued with symptoms after the second procedure. We proposed a Remeex-system. She didn't have uretha hipermobility. The surgery was succesful and currently she is asymptomatic.

The third one, had a TOT procedure two years before. She continues with urine stress incontinence without uretha hipermobility. We also proposed a Remeex-system. The surgery was succesful and currently she is asymptomatic.

\section{Conclusions:}

The choice of the appropiate surgical approach after failure of the initial technique should be individualized. It is always important to differentiate the presence of urethal hipermobility or intrinsic urethral dysfuntion, because in the latter case, the Remeex-System would have a clear indication.

The approach of recurrent SUI after TOT procedure is a therapeutic challenge in Soil Pelvix consultation. The incorporation of new surgical techniques is an important advance in the current management. 